\title{
La legitimidad desde el Parlamento Andino como órgano deliberante de la CAN ${ }^{*}$
}

\section{Legitimacy from the Andean Parliament as a deliberative organ of the CAN}

\section{Carolina Blanco Alvarado ${ }^{* *}$}

Fecha de recepción: 5 de julio de 2018 Fecha de aprobación: 20 de agosto de 2018

\section{RESUMEN}

Un rápido vistazo a la marcha del proceso de integración andino en los últimos años nos permite apreciar importantes avances relacionados con su futuro, como su reforzamiento institucional con la conversión del Pacto Andino en la Comunidad Andina, la coordinación de los organismos de la integración dentro del SAI (Sistema Andino de Integración), el desarrollo de la noción de integración integral y la elección directa por sufragio universal de los integrantes del Parlamento Andino.

La enumeración anterior, limitada a algunos de los elementos más relevantes de la labor de integración andina, nos indica la responsabilidad, la trascendencia, el alcance y los desafíos que tiene todavía por delante tal proceso.

* $\quad$ El artículo es producto del proyecto de investigación "El principio de reciprocidad de la contratación estatal en el marco de la Comunidad Andina (CAN)", gestionado y financiado por la Universidad Santo Tomás, Bogotá. Citar como Blanco Alvarado, C. (2019). La legitimidad desde el Parlamento Andino como órgano deliberante de la CAN. Via Inveniendi et Iudicandi, 15(2), 191-205. DOI: https://doi.org/10.15332/19090528.5047

** Ph. D. en Derecho, Universidad Santo Tomás, Bogotá. Magíster en Derechos Fundamentales, Universidad Carlos III, Madrid. Especialista en Derecho Constitucional, Centro de Estudios Políticos y Constitucionales, Madrid. Especialista en Derecho Administrativo, Universidad del Rosario, Bogotá. Docente e investigadora de la Universidad Santo Tomás, Bogotá, Colombia. Correo electrónico: ruthblanco@usantotomas.edu.co. Orcid: https://orcid.org/0000-0002-1354-4272. 
En ese contexto se pretende adelantar una aproximación a la crisis de legitimidad del proceso andino de integración, y un análisis del papel que para superarla debe cumplir el Parlamento Andino.

Palabras clave: integración andina, Parlamento Andino, democracia, legitimidad.

\section{Abstract}

A quick glance on the progress of the Andean integration process in recent years, allows us to appreciate the signaling of important advances related to the future of the process, such as its institutional reinforcement in the conversion of the Andean Pact into the Andean Community, the coordination of the integration agencies within the Andean integration system (SAI), the reception within the integration process of the notion of "integral integration" and the direct election by universal suffrage of the members of the Andean Parliament.

The enumeration above, which is limited to some of the most relevant elements of the work that is addressing Andean integration, indicates the responsibility, transcendence, scope and challenges that the Andean integration process still faces.

Under this contextualization, an approximation to the crisis of legitimacy of the Andean integration process is sought, and an analysis of the role that the Andean Parliament must fulfill in this regard to overcome it.

Keywords: Andean integration, Andean Parliament, democracy, legitimacy. 


\section{INTRODUCCIÓN}

Sin lugar a dudas el fenómeno de la globalización ha fortalecido los procesos de integración (Garzón, 2013), y dentro de ellos el de la CAN (Comunidad Andina). Al respecto es importante anotar que los cambios en los escenarios político, jurídico, social y económico experimentados a nivel mundial en los últimos años han acrecentado la interdependencia de los Estados. Lo anterior propicia en cada uno de estos la adopción de una nueva agenda internacional en la que los procesos de integración, en sentido multidimensional, adquieren una importancia muy significativa (Guarín y Aldana, 2016).

Una de las más representativas manifestaciones de la integración en Sudamérica ha sido la CAN, que desde su creación consideró la necesidad de involucrar a la población andina. Es por lo anterior que el Acuerdo de Cartagena desde el art. 1, prevé, dentro de los objetivos principales del proceso andino de integración,

la promoción y el desarrollo equilibrado y armónico de los países miembros en condiciones de equidad, mediante la integración y la cooperación económica y social; manteniendo como directriz el mejoramiento persistente en el nivel de vida de los habitantes de la región.

Desde 2007, la Comunidad Andina impulsó una transformación en la concepción y proyección del proceso, y decidió promover una integración integral que buscara un acercamiento mas equilibrado entre los aspectos sociales, económicos, políticos y jurídicos (Gonzales, 2009, p. 9). Es en este sentido que la integración andina debe trascender del plano económico a un escenario multidimensional de contenidos políticos, sociales y jurídicos. Bajo esta premisa el Parlamento Andino "ha concentrado su atención en torno a dos desafíos fundamentales: la integración social y la participación ciudadana; aspectos ciertamente relevantes para arraigar socialmente el proyecto integracionista con legitimidad" (Parlamento Andino, 1995, p. 5). 
Para Barco,

la política exterior de los Estados andinos, como política de Estado, se guía por el compromiso de proteger y preservar el interés nacional; buscando una mejor inserción de estos países en el mundo. Es por lo anterior que las relaciones de los Estados andinos con la comunidad internacional deben estar determinadas, entre otros factores, por procesos políticos y sociales de integración. (Barco, 2008, p. 44)

Es por ello que las constituciones de los países andinos prevén la necesidad de contextualizar las relaciones internacionales dentro del marco del proceso andino de integración en su gradual persecución de intereses comunitarios.

La aspiración de la integración andina es el compromiso de los Estados de la región de consolidar intereses o valores comunes y la obligación de subordinación de los intereses nacionales a aquellos. Ese compromiso debe ser liderado por un poder independiente, esto es, distinto del poder de los Estados participantes en el proceso de integración. Es bajo el citado contexto que el Parlamento Andino adquiere importancia como órgano capaz de liderar la consolidación y eficacia de la unidad entre los países andinos.

\section{Contextualización del Parlamento Andino}

De conformidad con el ex parlamentario andino Mariategui,

el proceso de integración subregional andino se origina el 16 de agosto de 1966, con la suscripción de la Declaración de Bogotá, firmada por Carlos Lleras Restrepo, presidente de Colombia; Eduardo Frei Montalva, presidente de Chile y Raúl Leoni, presidente de Venezuela; así como por Galo Plaza, representante de Clemente Yerovi Indaburu, presidente de Ecuador y Fernando Schwalb López-Aldana, representante de Fernando Belaúnde Terry, presidente del Perú. (Mariategui, 2009, p. 4) 
La finalidad era la alianza de los países andinos en aras de promover su desarrollo económico y social (Quiroz, 2014). Como cualquier proceso de integración, y atendiendo lo dispuesto por el doctrinante Tremps,

reclama la estructuración y puesta en funcionamiento de una organización administrativa y técnica, y determina la creación de autoridades comunitarias, con específicas competencias, y del mismo modo, la formulación de un sistema normativo con el vigor y la eficacia requeridos para que aquellas puedan regular directamente las cuestiones atinentes en materia de integración y la conducta de los países comprometidos y de sus habitantes, sin tener que recurrir a los procedimientos ordinarios del derecho interno de cada país. (1994, p. 39)

Como parte de esa perspectiva fue creado el Parlamento Andino, órgano comunitario deliberante de la CAN, de conformidad con las siguientes atribuciones asignadas por el art. 43 del Acuerdo de Cartagena:

a) Participar en la promoción y orientación del proceso de la integración subregional andina, con miras a la consolidación de la integración latinoamericana. b) Examinar la marcha del proceso de la integración subregional andina y el cumplimiento de sus objetivos, requiriendo para ello información periódica a los órganos e instituciones del sistema. c) Formular recomendaciones sobre los proyectos de presupuesto anual de los órganos e instituciones del sistema que se constituyen con las contribuciones directas de los países miembros. d) Sugerir a los órganos e instituciones del sistema las acciones o decisiones que tengan por objeto o efecto la adopción de modificaciones, ajustes o nuevos lineamientos generales con relación a los objetivos programáticos y a la estructura institucional del sistema. e) Participar en la generación normativa del proceso mediante sugerencias a los órganos del sistema de proyectos de normas sobre temas de interés común, para su incorporación en el ordenamiento jurídico de la Comunidad Andina. f) Promover la armonización de las legislaciones de los países miembros. g) Promover relaciones de cooperación y coordinación con los parlamentos de los países miembros, los órganos e instituciones del sistema, así como con los órganos parlamentarios de integración o cooperación de terceros países. 


\section{LA CRISIS DE LEGITIMIDAD EN LA CAN}

La integración andina ha evidenciado un déficit democrático, lo que no le ha permitido avanzar frente a los objetivos de la CAN. Para el efecto, dicho déficit se ha considerado como la incapacidad del proceso para integrar a la sociedad civil (Prieto, 2008). Lo anterior ocurre entre otros aspectos porque en el ámbito andino encontramos regímenes fuertemente presidencialistas, que juegan un rol determinante en la integración andina (Barragán, 2016). Dicho de otro modo, los Ejecutivos de los Estados andinos de alguna manera están absorbiendo todas las atribuciones de gestión del proceso, ejerciéndolas en virtud de su competencia de manejar las relaciones internacionales (Humbarita, 2015). Esta última función debe ser realizada por un órgano autónomo fuerte, con capacidad vinculante en sus pronunciamientos y que garantice la debida aplicación de las directrices integracionistas, lo cual solamente podrá darse cuando el Parlamento Andino goce de la legitimidad que le otorga tener miembros designados por vía de elección popular (Blanco, 2014).

El déficit democrático en el proceso andino de integración se refleja en la inexistencia de espacios deliberativos de decisión (González, 2016). Eso genera falta de participación activa tanto de los organismos del SAI como del pueblo andino, participación que de conformidad con la normatividad internacional y los propósitos de su creación debería ser promovida por el Parlamento Andino. Otras manifestaciones de tal déficit son la concentración de poder en los órganos intergubernamentales del SAI (Consejo Presidencial Andino y Consejo de Ministros de Relaciones Exteriores) y la reducción de funciones y autonomía de los órganos de carácter comunitario (Parlamento Andino y Tribunal de Justicia de la Comunidad Andina). Muestra de ello es que el Parlamento Andino en la práctica, a pesar de ser el órgano fundamental del involucramiento de la sociedad civil, tiene una función básicamente declarativa y deliberativa sin ningún poder decisorio y de carácter vinculante, a pesar de que normativamente está tipificado como un órgano capacitado para emitir decisiones comunitarias de ese tipo en la subregión (Patarroyo y Benavides, 2014).

La doctrina ha evidenciado que son casi inexistentes en la CAN las demandas por parte de los actores del nivel nacional sobre la democratización de los procesos de 
integración (Guarín, 2015). Según Prieto, eso “podría explicar en parte la escasez de espacios institucionales de participación en el nivel de la subregión” (2008, p. 20).

Los órganos que toman las decisiones de importancia en la CAN están compuestos por representantes de los intereses de cada Estado miembro. Como consecuencia de ello, se considera que tales delegados representan a los gobiernos nacionales (Saidiza y Carvajal, 2016). En este sentido resulta procedente afirmar que se trata de decisiones de gobierno, que no implican una diferencia significativa frente a las decisiones tomadas por esos mismos gobiernos a nivel interno (Prieto, 2008).

Por todo lo anterior se considera necesario que el proceso andino de integración se enmarque dentro de las políticas nacionales de Estado, mas no de gobierno, como quiera que durante sus ańos de vigencia la CAN se ha visto influenciada y obstaculizada por la orientación que cada gobierno otorga a su política exterior (Vélez, 2009).

La integración no es un proceso exclusivamente intergubernamental o interestatal. Por el contrario, necesita el concurso activo de los actores políticos y de la sociedad civil. Por eso resulta procedente afirmar que la participación de la ciudadanía es un factor de legitimidad y una condición de éxito pues si solo participan los gobiernos, el proceso integrador no evoluciona, y consecuentemente no se consolida.

Ahora bien, los países andinos, a pesar de haber empezado a construir la integración hace cuatro décadas, aún no se han preparado como Estados para trazar su norte integracionista. Un análisis somero de las estructuras de los ministerios de Relaciones Exteriores de tales países muestra que en todos siempre hay una instancia encargada de asuntos de soberanía territorial y nunca una de asuntos de integración, o al menos esta última esta relegada a una instancia de menor jerarquía. Lo anterior pone de manifiesto que seguimos atados a un concepto de soberanía que en el marco del siglo XXI e inmersos en un mundo globalizado resulta cuando menos anacrónico. Hoy por hoy la interacción de los Estados en el escenario internacional obedece a nuevos parámetros de cooperación en todos los ámbitos, que resultan fundamentales para enfrentar exitosamente los retos en los ámbitos político, económico, social y jurídico. Así, concebir a los Estados solo de las fronteras hacia adentro resulta fuera de contexto 
e incluso perjudicial. La soberanía debe evidenciar un nuevo entendimiento en el cual los Estados, sin desconocer sus límites políticos y jurídicos de interacción en la comunidad internacional, aprendan a relacionarse de sus fronteras hacia afuera, y sus estructuras internas deben evolucionar en ese mismo sentido (Montero, 2009).

En concordancia con lo anterior, es pertinente hacer referencia a lo dispuesto por el parlamentario andino Juan Mariategui, quien manifiesta que una de las mayores debilidades de la CAN radica en su carácter intergubernamental. El ámbito de elaboración de normativas se encuentra concentrado en dos órganos que dependen directamente de los poderes ejecutivos de los países miembros, el Consejo Andino de Ministros de Relaciones Exteriores y la Comisión de la Comunidad Andina (constituida esta última por los ministros de Comercio Exterior). Estos dos órganos generan las más importantes normas que produce la CAN, es decir, las decisiones, tal y como lo establece el Acuerdo de Cartagena en sus arts. 17 y 21.

En el proceso de generación de las normas andinas el Parlamento Andino no tiene facultades de control o supervisión puesto que tanto el Acuerdo de Cartagena (art. 42) como su Tratado Constitutivo (art. 1) lo definen únicamente como órgano deliberante. Su atribución en ese sentido es participar en el proceso normativo sugiriendo a los órganos del sistema proyectos de normas sobre temas de interés común para su incorporación en el ordenamiento jurídico de la CAN, sin capacidad de crear normativa supranacional de carácter vinculante, a diferencia de las decisiones que emiten la Comisión o el Consejo de Ministros de Relaciones Exteriores. Es importante anotar que al depender la generación de normas comunitarias de los órganos intergubernamentales, automáticamente esta acción queda sujeta a lo que acontece en los países miembros en lo que respecta al desenvolvimiento de sus actores económicos y políticos y a la marańa de intereses sectoriales en juego. Por lo anterior, el Parlamento Andino no debe ser únicamente un órgano deliberante, sino también protagonista en el proceso de generación de normas; en otras palabras, convertirse en constituyente andino con capacidad de codecisión (Mariategui, 2009).

Adicional a lo dispuesto anteriormente es importante anotar que otra causal de la crisis de legitimidad del proceso andino de integración es el incumplimiento de algunos requisitos básicos de los Estados democráticos en los países andinos. Cuando 
los Estados miembros presentan fragilidades o inconsistencias en sus propios sistemas democráticos ello se refleja en el funcionamiento del sistema comunitario y en el alejamiento de los objetivos y propósitos de la CAN (Paradisi, 2005).

\section{Cómo SUPERAR LA CRISIS DE LEGITIMIDAD DEL PROCESO ANDINO DE INTEGRACIÓN}

En aras de aproximarnos a una posible solución al problema diagnosticado es necesario romper el aislamiento de las instituciones encargadas de promover la integración andina identificando en forma conjunta metas y estrategias y levantando una agenda que responda a las necesidades de los pueblos y dote de legitimidad al proceso (González, 2014). La efectividad de la citada propuesta depende fundamentalmente de la consolidación jurídica y política en cada uno de los Estados que conforman la CAN de la elección directa de parlamentarios andinos.

A pesar de que la mayoría de los Estados andinos han procedido con la reglamentación de la elección directa de parlamentarios andinos, es preciso puntualizar algunas circunstancias que no han permitido la consolidación de dicho proceso de elección:

1) La inexistencia del un régimen electoral uniforme en los países andinos, que regule los referidos procedimientos electorales. La CAN aspira a consolidar un régimen electoral uniforme para la elección de todos sus representantes en el Parlamento Andino independientemente de su país de origen, de conformidad con el Tratado de Elecciones Directas Universales de Parlamentarios Andinos. Pero mientras se establece ese régimen el sistema de elección de los representantes ante el Parlamento Andino se regirá por la legislación electoral interna de cada Estado. Para impulsar el cambio se potenció el papel del CEA (Consejo Electoral Andino, insertado al SAI), mediante la Decisión 551 de la Comunidad Andina ${ }^{1}$, como órgano encargado de liderar la proyección del referido tratado. Dicho Consejo tiene entre sus atribuciones: emitir

1 La Decisión 551 de 2003 emitida por la CAN consagra la creación del Consejo Electoral Andino como instrumento del Sistema Andino de Integración. Estará conformado por los titulares de los órganos electorales de los países miembros de la Comunidad Andina. 
opinión ante el Consejo Andino de Ministros de Relaciones Exteriores y la Secretaría General de la Comunidad Andina en los ámbitos de su competencia cuando así se lo requieran; elaborar y recomendar la aprobación del Estatuto Electoral Andino y sus reformas; auspiciar mecanismos de cooperación horizontal y asistencia técnica entre los órganos electorales de la región; supervisar y observar los procesos de elección directa de los parlamentarios andinos; sugerir medidas para el perfeccionamiento de la legislación electoral interna relacionada con la elección de los parlamentarios andinos en cada país miembro; entre otras (Palomares y Calonje, 2015).

2) La no superación del déficit democrático que atraviesa el proceso andino de integración. Si bien la elección directa de los parlamentarios comunitarios es un objetivo deseado y deseable, es pertinente repetir que en el ámbito andino encontramos regímenes fuertemente presidencialistas, lo que hace que los congresos nacionales jueguen un rol ciertamente secundario. Por lo anterior, los Ejecutivos de los Estados andinos van a seguir jugando un rol determinante en el proceso de integración andina, anclado en la institucionalidad presidencialista de la región (Blanco, 2015), absorbiendo todas las atribuciones de gestión del proceso de integración andino y ejerciéndolas en virtud de su competencia de manejar las relaciones internacionales. En otras palabras, se aprecia que dentro del contexto de los Estados andinos se presenta una gran concentración del proceso integracionista en manos de las autoridades ejecutivas, que diseñan políticas, las implementan y ejercen el control de su aplicación ante la debilidad de otro ente evaluador que lo haga (Rodríguez, 2014).

\section{Propuestas de solución frente a la CRISIS De legitimidad en la CAN desde el Parlamento Andino}

1) El Parlamento Andino debe adelantar todas las gestiones necesarias para coordinar la consolidación política y jurídica del régimen electoral uniforme que regule el procedimiento electoral andino. Lo anterior con la finalidad de poner en marcha el proyecto que conduzca a sentar las bases normativas de las elecciones directas y universales de los parlamentarios andinos, lo cual no es mas que el cumplimiento del Tratado de Elecciones Directas Universales, el cual establece la obligación de los 
Estados integrantes de la CAN de gestionar un régimen electoral uniforme. Para el efecto, se debe potenciar el papel del CEA como órgano encargado de liderar la proyección del documento referenciado.

2) El proceso de integración andino requiere de la consolidación de una comunidad de Estados democrática e intercultural, que reconozca una ciudadanía andina activa, basada en la participación política y cívica, y que vele por un conjunto de libertades, derechos y garantías que se sumen y confieran un valor añadido a las ciudadanías nacionales. En este sentido, es el Parlamento Andino el órgano encargado de liderar el proceso de reconocimiento político y jurídico de la ciudadanía andina dentro de los Estados que conforman la CAN (Blanco, 2013).

La ciudadanía andina asegura sentido de pertenencia a la CAN y afianzamiento de una identidad andina, basada en el reconocimiento de valores democráticos e interculturales, el respeto de los derechos humanos, la no discriminación por razón de la nacionalidad y un conjunto de libertades y derechos sociales, ambientales, culturales y económicos, que los nacionales de los países miembros pueden ejercer en un territorio integrado (Rodríguez, 2016).

Dicha ciudadanía tendría las siguientes características:

Será ciudadana de la Comunidad Andina toda persona que ostente la nacionalidad de un Estado miembro. La nacionalidad se concederá y se reconocerá en concordancia al derecho nacional de los Estados miembros.

b) La ciudadanía andina es complementaria y no sustitutiva de la ciudadanía nacional, ya que no la elimina. La ciudadanía andina coexiste y presupone la ciudadanía de un Estado miembro, es decir la nacionalidad de un Estado miembro es condición sine qua non para ser ciudadano de la Comunidad Andina.

c) La ciudadanía andina significa que las personas no pueden ser objeto de discriminaciones en el ejercicio de sus derechos ni por las autoridades públicas 
(administrativa o judicial) ni por otros particulares en cualquier Estado miembro.

d) Todo ciudadano de la Comunidad Andina que resida en un Estado miembro del que no sea nacional tendrá derecho de elegir y ser elegido en las elecciones al Parlamento Andino en el Estado miembro en el que resida, en las mismas condiciones que los nacionales de dicho Estado.

e) Todo ciudadano de la Comunidad Andina tendrá derecho a circular y residir libremente en el territorio de los Estados miembros, con sujeción a las disposiciones adoptadas para su aplicación. (Sánchez, 2009, p. 310)

\section{ConClusiones}

El proceso andino de integración es democrático, y como consecuencia de ello la consolidación de la elección directa y universal de parlamentarios andinos es el principal mecanismo para fortalecer el proceso regional de integración y ayudar a superar su crisis de legitimidad.

El Parlamento Andino debe ser el espacio obligado para el debate democrático en el contexto de la supranacionalidad. Esto implica que, como órgano deliberante, sea una instancia para el encuentro de los intereses nacionales y subregionales en el marco de la discusión democrática.

La ausencia de participación política ha dado lugar a que el proceso andino de integración se lidere a un nivel gubernamental, sin que se haya podido trascender a la integración entre los pueblos. El fortalecimiento del Parlamento Andino puede determinar el avance de tal proceso, si se basa en políticas públicas interestatales y no intergubernamentales que acojan el interés de los ciudadanos andinos.

En la Comunidad Andina es necesario procurar el fortalecimiento de las instituciones desde una perspectiva verdaderamente democrática. 


\section{REFERENCIAS}

Barco, C. (2008). Aportes para el estudio de la politica exterior. Bogotá: Academia Diplomática de San Carlos.

Barragán, D. (2016). La construcción de la mentalidad democrática como necesidad en el posconflicto. Via Inveniendi et Iudicandi, 11(1), 37-57.

Blanco, C. (2013). Algunas propuestas frente a la crisis de la legitimidad de la Comunidad Andina (CAN). Análisis Político, 26(78), 141-155.

Blanco, C. (2014). La crisis de la Comunidad Andina. Revista de Derecho, 42, 1-32.

Blanco, C. (2015). La influencia positiva de la CAN en la descentralización territorial colombiana. Opinión Jurídica, 14(27), 161-174.

Garzón, E. (2013). Globalización del derecho, fetichismo legal, el velo de los derechos humanos. Verba Iuris, 30, 169-181.

Gonzales, C. (2009). Agenda social andina: ¿una realidad? Rescate del Pensamiento Latinoamericano, 6, 45-76.

González, O. (2014). La Corte Constitucional como agente del campo jurídico colombiano: la omisión legislativa de principios constitucionales. Revista Iusta, 41, 123-137.

González, O. (2016). La omisión legislativa como hecho generador de la responsabilidad patrimonial del Estado. Verba Iuris, 11(35), 41-63.

Guarín, E. (2015). Una aproximación filosófico-jurídica al sentido de la expresión: "Realización efectiva de los derechos”. Verba Iuris, 34, 147-159.

Guarín, E. A. y Aldana, J. (2016). Estado jurisdiccional y bien común. Verba Iuris, 11(36), $13-26$. 
Humbarita, J. (2015). Derecho constitucional hispanoamericano frente a la realidad institucional, manifiesta divergencia. Revista Iusta, 43, 91-118.

Mariategui, J. (2009). Globalizacion e integracion latinoamericana. Lima: Parlamento Andino.

Montero, P. (2009). El proceso andino de integración. Rescate del Pensamiento Latinoamericano, 5, 23-45

Palomares, J. y Calonje, N. (2015). Tratados de libre comercio Colombia-Asia: cuestión preliminar y perfiles de negociación. Revista Iusta, 43(1), 17-41.

Paradisi, J. (2005). La inestabilidad politica de los países andinos como uno de los obstáculos para la profundización del proceso de integración. Recuperado de http://vlex.com/ $\mathrm{vid} / 44666468$

Parlamento Andino (1995). Memorias del XI periodo extraordinario de sesiones. Bogotá: Parlamento Andino.

Patarroyo, S. y Benavides, P. (2014). Rupturas asignificantes: revisiones críticas en torno al derecho. Via Inveniendi et Iudicandi, 9(1), 7-31.

Prieto, C. (2008). Integración y democracia: aspectos socio-politicos del regionalismo en Suramérica. Bogotá: Universidad Nacional de Colombia.

Quiroz, M. (2014). Acercamiento a las “oposiciones paradigmáticas” entre neoconstitucionalismo y positivismo jurídico. Revista Iusta, 41(2), 77-97.

Rodríguez, A. (2014). Indicadores de constitucionalidad de las políticas públicas: enfoque de gestión de derechos. Via Inveniendi et Iudicandi, 9(2), 135-175.

Rodríguez, E. (2016). El pasaje del Estado y el derecho a la postmodernidad. Via Inveniendi et Iudicandi, 11(2), 11-37. 
Saidiza, H. y Carvajal, J. (2016). Crisis del Estado de derecho en Colombia: un análisis desde la perspectiva de la legislación penal. Revista Iusta, 44(1), 17-39.

Sánchez, T. D. (2009). Participación ciudadana en la CAN. Visión académica. Bogotá: Universidad de San Buenaventura.

Tremps, P. (1994). Constitución española y Comunidad Europea. Madrid: Ariel.

Vélez, R. (2009). El panorama actual de la Comunidad Andina. Rescate del Pensamiento Latinoamericano, 6, 67-87. 